Research Article

\title{
Shock Reduction Technique on Thin Plate Structure by Wave Refraction Using an Elastic Patch
}

\author{
Dae-Hyun Hwang and Jae-Hung Han \\ Department of Aerospace Engineering, KAIST, Daejeon 34141, Republic of Korea \\ Correspondence should be addressed to Jae-Hung Han; jaehunghan@kaist.ac.kr
}

Received 20 August 2021; Revised 1 November 2021; Accepted 19 November 2021; Published 6 December 2021

Academic Editor: Arcanjo Lenzi

Copyright (c) 2021 Dae-Hyun Hwang and Jae-Hung Han. This is an open access article distributed under the Creative Commons Attribution License, which permits unrestricted use, distribution, and reproduction in any medium, provided the original work is properly cited.

\begin{abstract}
In general, bending waves transfer the largest portion of shock energy in a plate-like structure. This study proposes a new shock reduction method using an elastic patch designed to defocus the bending waves through the refraction of the waves so that it is possible to effectively reduce the propagating shock for a certain target area. Elastic patches of three different shapes were considered. The shock reduction performance of these patches was analytically, numerically, and experimentally investigated and compared. All results consistently showed that attached patches can effectively reduce passing waves for areas behind patches. Therefore, utilizing the proposed methods, we can reduce the transient shock response at certain target areas of various practical structures without degradation of structural stiffness or strength simply by bonding with an elastic patch.
\end{abstract}

\section{Introduction}

There have been many efforts to reduce structural shocks propagating as elastic waves in various engineering applications, including aerospace structures in which thin platelike structures are often used as basic elements. The application of shock absorbers is the most common method to reduce shock propagation. A shock absorber is installed at the interface of a structure mounted on a base plate to attenuate the shock transmitted along the plate. An acoustic absorber made of various foams is applied to the surface to reduce acoustic noise generated from the plate structure. Acoustic noise induced by plate structures tends to be dominated by bending waves [1]. In general, the antisymmetric mode of Lamb waves, also called bending waves, transfers the largest portion of energy. Over much of the audio-frequency range, bending waves in thin plates have the lowest mechanical impedance of the structure-borne waves; as a result, they tend to be most strongly excited by transient forces [1].

Shock isolators and absorbers have been investigated and widely used to attenuate shock transmission to subsystems. Jeong et al. $[2,3]$ developed a frequency tunable vibration and shock isolator using a shape memory alloy (SMA) wire actuator for the application of pyroshock attenuation in launch vehicles and satellites. Youn et al. [4] introduced a three-axis hybrid mesh isolator using the pseudoelasticity of an SMA wire to attenuate pyroshock and vibration transmitted to electronic components. They proved that there was significant shock energy absorption by the pseudoelastic effect of the SMA wire. Park et al. [5] proposed a sandwich panel insert to achieve enhanced shock attenuation without a shock isolator, with potential applications including connecting parts or mounting devices on panels.

Since 2000, many investigations have been conducted to manipulate elastic waves in plates. There are various wave manipulation methods, such as the use of an acoustic black hole $(\mathrm{ABH})$ or of metastructures. The acoustic black hole effect, introduced by Mironov [6], can achieve almost perfect absorption of the incident wave energy. The main principle is a linear or higher-order power-law decrease in the velocity of the incident wave. The $\mathrm{ABH}$ effect has been investigated mainly for bending waves in thin plates [7]. The $\mathrm{ABH}$ technique shows good performance in absorbing wave energy; however, there is a difficulty in that the already thinplate structure needs to be made thinner or needs cut-out 
holes, which cause degradation of the structural stiffness and strength for some practical applications. Several studies on the wave manipulation method based on metamaterials have also been conducted. Zhu et al. [8] presented an elastic metamaterial with chiral microstructures to achieve negative refraction of elastic waves. Dubois et al. [9] demonstrated broadband focusing of elastic waves at $10 \mathrm{kHz}$ in a thin plate with a 45-degree tilted square array of circular air holes, which cause a negative refraction effect by changing the relative thickness. $\mathrm{Li}$ et al. [10] proposed a sharp bending scheme for acoustic waves by truncating the sonic crystals. In this study, an all-angle and wide-band $90^{\circ}$ bending waveguide was demonstrated at a wide range of incident angles.

Recently, there have been a few efforts to control bending wave and noise in a thin plate-like structure without cut holes. Darabi et al. [11] and Zareei et al. [12] proposed a wave-bending structure in a thin plate by smoothly varying the bending stiffness of the plate and phase velocity. The proposed structure does not require bandgaps; therefore, it is frequency-independent and able to guide bending waves along arbitrary curvy trajectories or effectively focusing the bending waves. Li et al. [13] and Qi et al. [14] investigated the internal noise reduction of cylindrical shell structure using the noise control method of laying melamine-foam layer to reduce the harsh mid-low frequency noise of rocket fairing. They conducted experiments and numerical analysis on aluminium and aluminium/melamine-foam cylindrical shells and obtained corresponding internal noise response spectrograms.

As with the $\mathrm{ABH}$ technique, however, the metamaterials or machining on a thin plate have limitations in terms of degradation of structural rigidity and strength of the plate structures. Recently, a few studies have been conducted on a technique of refracting bending waves simply by attaching a metal slice to the plate without indentation or hole processing. The velocity of waves in a thin plate structure is determined by the material properties and the thickness of the plate. In wave theory, waves propagating from one medium to the other are refracted by the propagation velocity difference, and this refraction phenomenon is also applicable to bending waves on plates [15]. A numerical and experimental study conducted by Tian and $\mathrm{Yu}$ [16] showed that an A0-mode wavefront could be focused on the desired focal point using a planoconcave aspherical lens by changing the effective plate thickness using lenses bonded on plates. Kudela and Ostachowicz [17] also showed that a planoconcave aspherical lens attached to the plate can change the effective plate thickness and can be used to focus the A0 mode of Lamb waves. Tol et al. [18] introduced a study on 3D-printed Gradient-Index Phononic Crystal Lens bonded on a metallic host plate in order to enhance energy harvesting by focusing the elastic wave. The gradient refractive index was achieved by designing stub heights with the hyperbolic distribution. Darabi et al. [19] studied Broadband cloaking of bending waves by a flexural cloaking structure using only a single material composed of homogeneous and isotropic layers and experimentally showed the near-ideal broadband cloak in thin plates.
In this study, a shock reduction method is investigated using various shapes of elastic patches to defocus the bending waves through the refraction of the waves. Elastic patches of three different shapes were considered to refract and reduce the transient bending waves; the shock reduction performances of those patches were investigated analytically, numerically, and experimentally. The proposed method uses elastic patches, which can be easily applied to practical structures to refract the bending waves, reducing them at selected regions without any degradation of the structural stiffness or strength. The proposed method has potential applications in protecting sensitive components such as sensors from intensive shock and noise in plate-like structures, in aerospace, automobiles, and constructions.

\section{Refraction of Bending Waves}

According to the Kirchhoff-Love plate theory (classical plate theory), the deflection of the middle plane of a plate $w(x, y, t)$ for an isotropic plate is governed by [20]

$$
D \nabla^{4} w(x, y, t)+\rho h \frac{\partial^{2} w(x, y, t)}{\partial t^{2}}=0,
$$

where $D$ is the bending stiffness of the plate, $\rho$ is the volume mass density, $h$ is the thickness of the plate, and $\nabla$ is the Laplacian for $x$ and $y$. The propagation velocity of bending waves " $c$ " in the thin uniform plate is given by $[1,20]$

$$
c=\sqrt{\omega}\left(\frac{D}{m}\right)^{1 / 4}
$$

where $\omega$ is the phase frequency of bending waves and $m$ $(=\rho h)$ is mass per unit area.

When an elastic wave propagates from one solid to another solid, the following four quantities must be equal on both sides of the boundary [15]: the normal displacement, the tangential displacements, the normal stress, and the tangential stress. As shown in Figure 1, when vibrations of an incident bending wave are in the $x y$-plane with the incident angle of $\beta_{1}$, the wave reflects with the same angle and refracts at an angle of $\beta_{3}$. The deviation angle with respect to the direction of incident waves is defined as $\gamma$. In an infinite flat plate, bending waves generated by a concentrated transverse force spread out cylindrically from a source point [1]. According to Huygens's principle, the wavefront at any moment is on the envelope of a series of spherical wavelets spread out from points on the wavefront at a previous moment, and this principle is used to derive the sine law (Snell's law) relation $[1,15]$

$$
\frac{\sin \left(\beta_{1}\right)}{c_{A}}=\frac{\sin \left(\beta_{2}\right)}{c_{A}}=\frac{\sin \left(\beta_{3}\right)}{c_{B}},
$$

where $c_{A}$ and $c_{B}$ are the propagation velocities of the bending waves in the first plate and the second plate, respectively. That is to say, for a given pair of media, how much a wave is refracted is determined by the change in wave speed and the initial direction of the incident wavefronts.

By utilizing the refraction phenomenon of bending waves, it is possible to reduce the shock response propagated 


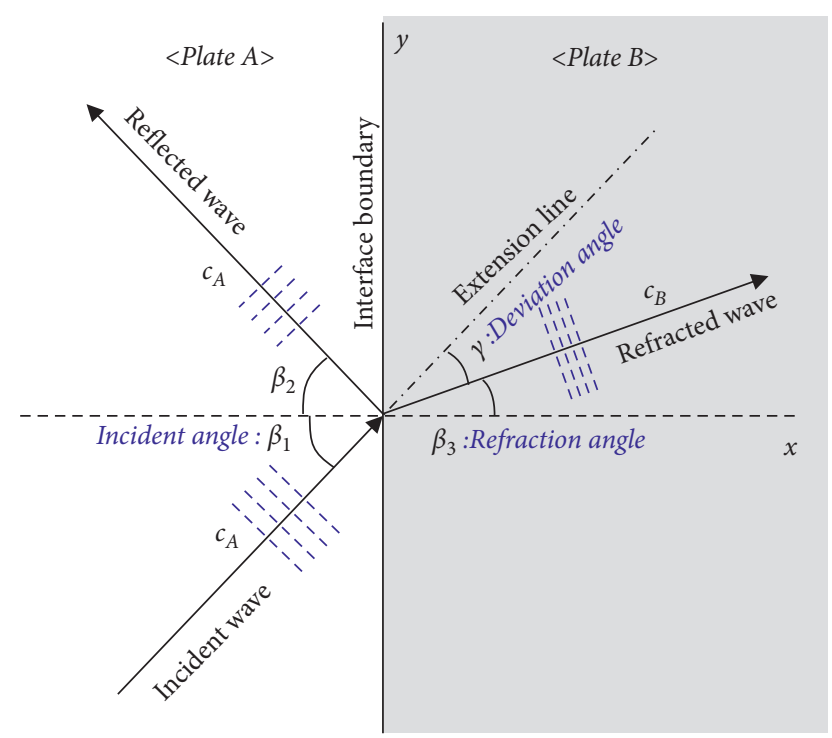

FIGURE 1: Reflection and refraction of incident distortion wave at the plane interface.

in a certain target area. Effective refraction of bending waves can be achieved simply by attaching a thin elastic patch to the host plate to change the bending stiffness. Figure 2 shows the concept of the shock reduction method proposed in this study. Numerical and experimental investigations to identify the shock reduction performance will be discussed in the next section.

\section{Effect of Patch Thickness on the Bending Wave Propagation}

3.1. Evaluation of the Bending Stiffness of Multilayered Beam Structure. As a preliminary study on the wave refraction by the elastic patch, theoretical, numerical, and experimental studies were conducted to identify the effect of patch thickness on the bending wave propagation in the laminated plates combined by adhesives.

The deformation of a plate assembly shown in Figure 3 consists of two parts: deformations due to bending moments, $w_{b}$, and deformations due to transverse forces, $w_{s}$. Therefore, a total deformation is a summation of those two deformations as $w=w_{b}+w_{s}$.

The location of the neutral axis, $e$, from the bottom surface of the host plate defined as $z=0$, is given by the coordinate system for which the first moment of area is zero when integrated over the entire cross section [21]:

$$
e=\frac{E_{p} t_{p} z_{p}+E_{a} t_{a} z_{a}+E_{h} t_{h} z_{h}}{E_{p} t_{p}+E_{a} t_{a}+E_{h} t_{h}},
$$

where $E$ and $\nu$ are Young's modulus and Poisson's ratio. The subscripts $p, a$, and $h$, respectively, denote the patch, the adhesive, and the host plate. $z_{p}, z_{a}$, and $z_{h}$ are the locations of the neutral axis of the patch, adhesive, and host plate, respectively. The effective bending stiffness of the plate assembly shown in Figure 3 can be explained using the sandwich plate theory [21].

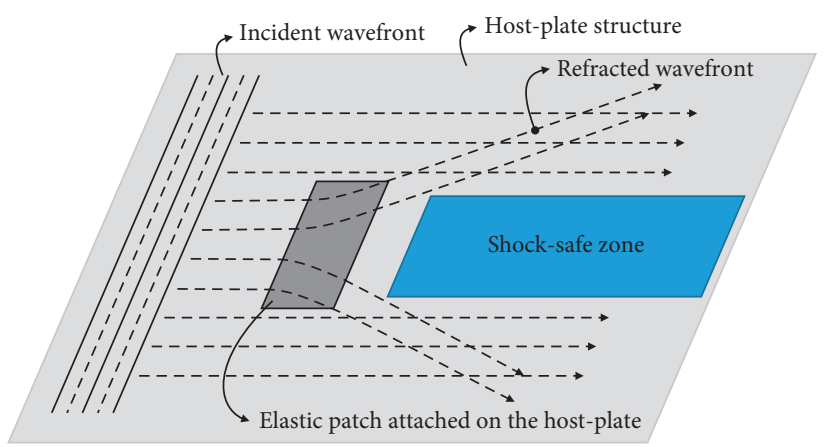

FIgURE 2: Conceptual diagram of proposed shock reduction method.

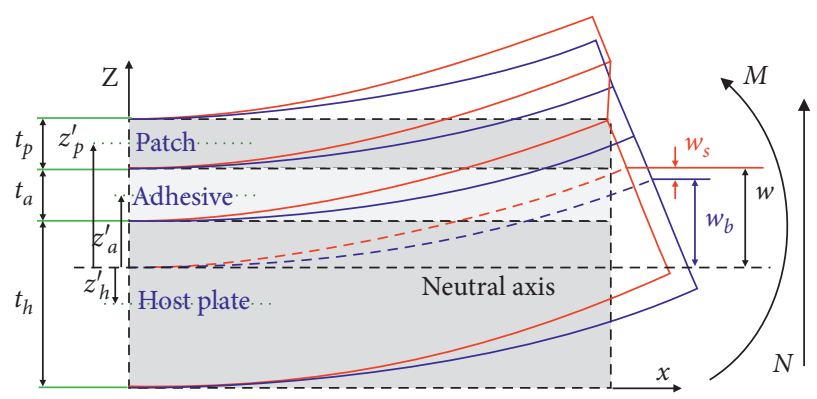

Figure 3: Deformation of a plate assembly with adhesive layer.

First, the constitutive relations for $2 \mathrm{D}$ orthotropic linear elastic materials are given as follows [21]:

$$
\left[\begin{array}{c}
\sigma_{x x} \\
\sigma_{z z} \\
\sigma_{z x}
\end{array}\right]=\left[\begin{array}{ccc}
C_{11} & C_{13} & 0 \\
C_{13} & C_{33} & 0 \\
0 & 0 & C_{55}
\end{array}\right]\left[\begin{array}{l}
\varepsilon_{x x} \\
\varepsilon_{z z} \\
\varepsilon_{z x}
\end{array}\right],
$$

where three assumptions with bending of thin face sheets can be considered as follows: (i) the thickness of each layer in the $z$-direction does not change; (ii) the in-plane normal stiffness of the core is small compared to that of the face sheet; that is, the core does not lengthen or compress in the $x$-direction; and (iii) the face sheet (the patch and the host-plate) follows the Euler-Bernoulli assumptions so that there is no $x z$-shear deformation in the face sheet, but the $x z$-shear deformation in the core is not neglected. Equations (6) and (7) summarize the described assumptions:

$$
\begin{gathered}
\sigma_{z z}^{p}=\sigma_{z x}^{p}=\sigma_{z z}^{h}=\sigma_{z x}^{h}=\sigma_{z x}^{h}=0, \\
\varepsilon_{z z}^{p}=\varepsilon_{z z}^{h}=\varepsilon_{z z}^{a}=\varepsilon_{z x}^{p}=\varepsilon_{z x}^{h}=0,
\end{gathered}
$$

where the superscripts $p, a$, and $h$, respectively, denote the patch, the adhesive, and the host plate. Based on the kinematics of the plate assembly shown in Figure 3 and the assumptions of the sandwich plate theory in equations (6) and (7), the stress-displacement relations of the patch, the host plate, and the adhesive can be derived as follows: 


$$
\begin{aligned}
& \sigma_{x x}^{p}=-(z-e) C_{11}^{p} \frac{\mathrm{d}^{2} w_{b}}{\mathrm{~d} x^{2}}-\left[z-\left(e+z_{p}^{\prime}\right)\right] C_{11}^{p} \frac{\mathrm{d}^{2} w_{s}}{\mathrm{~d} x^{2}}, \\
& \sigma_{x x}^{h}=-(z-e) C_{11}^{h} \frac{\mathrm{d}^{2} w_{b}}{\mathrm{~d} x^{2}}-\left[z-\left(e+z_{h}^{\prime}\right)\right] C_{11}^{h} \frac{\mathrm{d}^{2} w_{s}}{\mathrm{~d} x^{2}}, \\
& \sigma_{x x}^{a}=-(z-e) C_{11}^{a} \frac{\mathrm{d}^{2} w_{b}}{\mathrm{~d} x^{2}}-\left[z-\left(e+z_{a}^{\prime}\right)\right] C_{11}^{a} \frac{\mathrm{d}^{2} w_{s}}{\mathrm{~d} x^{2}} \\
& \sigma_{z x}^{a}=C_{55}^{a}\left(\frac{t_{a}+\left(t_{p}+t_{h}\right) / 2}{2 t_{a}}\right) \frac{\mathrm{d} w_{s}}{\mathrm{~d} x} .
\end{aligned}
$$

The resultant forces and moment relationships are given as

$$
\begin{aligned}
& N_{x x}^{p}=\int_{-t_{p} / 2}^{t_{p} / 2} \sigma_{x x}^{p} \mathrm{~d} z^{p}, \\
& M_{x x}^{p}=\int_{-t_{p} / 2}^{t_{p} / 2} z^{p} \sigma_{x x}^{p} \mathrm{~d} z^{p}, \\
& N_{x x}^{h}=\int_{-t_{h} / 2}^{t_{h} / 2} \sigma_{x x}^{h} \mathrm{~d} z^{h}, \\
& M_{x x}^{h}=\int_{-t_{h} / 2}^{t_{h} / 2} z^{h} \sigma_{x x}^{h} \mathrm{~d} z^{h}, \\
& N_{x x}^{a}=\int_{-t_{a} / 2}^{t_{a} / 2} \sigma_{x x}^{a} \mathrm{~d} z^{a}, \\
& M_{x x}^{a}=\int_{-t_{a} / 2}^{t_{a} / 2} z^{a} \sigma_{x x}^{a} \mathrm{~d} z^{a} .
\end{aligned}
$$

From the definition of bending stiffness, given in equation (10), assuming that the shear deformation of the plate is negligibly small and the moduli of the patch and host plate are sufficiently greater than that of the adhesive, the bending stiffness of the plate assembly is derived as equation (11):

$$
\begin{aligned}
& D=-\frac{M}{\mathrm{~d}^{2} w / \mathrm{d} x^{2}}, \\
& D=t_{p} z_{p}^{\prime 2} C_{11}^{p}+t_{h} z_{h}^{\prime 2} C_{11}^{h}+\frac{t_{p}^{3}}{12} C_{11}^{p}+\frac{t_{h}^{3}}{12} C_{11}^{h},
\end{aligned}
$$

where $z_{p}^{\prime}$ and $z_{h}^{\prime}$ are the location of the neutral axis of the patch and host plate with respect to the neutral axis of the assembly, respectively. Therefore, when a patch is attached to the host plate, the bending stiffness increases and the wave propagation speed tends to increase, as explained in equation (11).

3.2. Numerical Simulation on the Propagation Speed of the Bending Wave. To numerically validate the effect of the patch, a finite element model of a $2 \mathrm{D}$ plain-strain beam as shown in Figure 4 with a total length of $600 \mathrm{~mm}$ was established and analysis for the propagation of bending wave was performed. Figure 4 shows the magnified section of the beam. In order to generate a bending wave, a $10 \mathrm{kHz}$

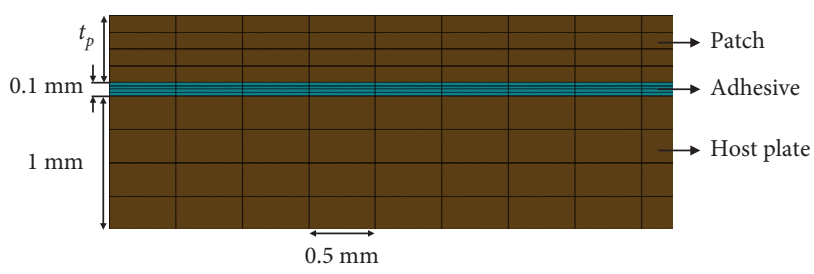

FIGURE 4: Finite element model of a part of the beam assembly with the adhesive layer.

sinusoidal velocity boundary condition of 4 -cycles in the vertical direction was applied to one end of the beam. The linear element with a size of $0.5 \mathrm{~mm}$ in length and 4-elements in thickness direction for each layer was used. It is known that the linear element has a shear locking phenomenon. However, the shear locking can be prevented by using at least 3 elements over the thickness, and also three layers of linear elements across the thickness are sufficient for bending analysis [22].

Aluminium was considered for the material of the patch and the host plate, and epoxy resin was considered as the adhesive material. The material properties are listed in Table 1.

3.3. Experimental Validation on the Propagation Speed of the Bending Wave. Figures 5 and 6 show a schematic diagram and the actual experimental setup to measure the wavelength depending on the patch thickness, respectively. The common host plate was made of aluminium with dimensions of $1000 \mathrm{~mm} \times 50 \mathrm{~mm} \times 1 \mathrm{~mm}$. To simulate the free edge boundary condition, the host plate was suspended by two polypropylene wires. The beam patches with various thicknesses are made of the same material and dimensions as the host plate. To measure the out-of-plane velocity response of the patch side, a Laser Doppler Vibrometer (LDV) mounted on a 2D stage system was used. A piezoelectric disk made of PIC255 [26] (piezoelectric ceramic material) with $10 \mathrm{~mm}$ diameter and $0.5 \mathrm{~mm}$ thickness was attached to the host plate. The waveform of the input voltage to the piezo disk actuators was set to a Hanning-windowed sinusoidal signal of three periods, with voltage amplitude from $0 \mathrm{~V}$ to $300 \mathrm{~V}$, amplified using a Trek-623B [27] piezoelectric driver. The velocity histories at each sensing point were measured with a sampling rate of $1 \mathrm{MHz}$ using a National Instruments PXIe-6366 [28] DAQ board.

Figure 7 shows wavenumbers evaluated by the numerical simulation, the analytical analysis, and the experiments; all three (the analytical, the numerical, and the experimental) results agree well.

\section{Shock Reduction by Wave Refraction through an Elastic Patch}

4.1. Experimental Structure. Figure 8 shows schematics of the configuration for the experimental object structure. The host plate is made of aluminium of dimensions $1200 \mathrm{~mm} \times 1200 \mathrm{~mm} \times 1 \mathrm{~mm}$ (thickness). The plane bending waves are generated from an array of 15 piezoelectric disk 
TABLe 1: Material properties of aluminium [23] and epoxy resin [24, 25].

\begin{tabular}{lcc}
\hline Material & $\begin{array}{c}\text { Host plate and patch } \\
\text { Aluminium }\end{array}$ & $\begin{array}{c}\text { Adhesive } \\
\text { Epoxy resin }\end{array}$ \\
\hline Density $\left(\mathrm{kg} / \mathrm{m}^{3}\right)$ & 2770 & 1160 \\
Young's modulus $(\mathrm{GPa})$ & 71 & 3.78 \\
Shear modulus $(\mathrm{GPa})$ & 26.7 & 1.4 \\
Poisson's ratio & 0.33 & 0.35 \\
\hline
\end{tabular}

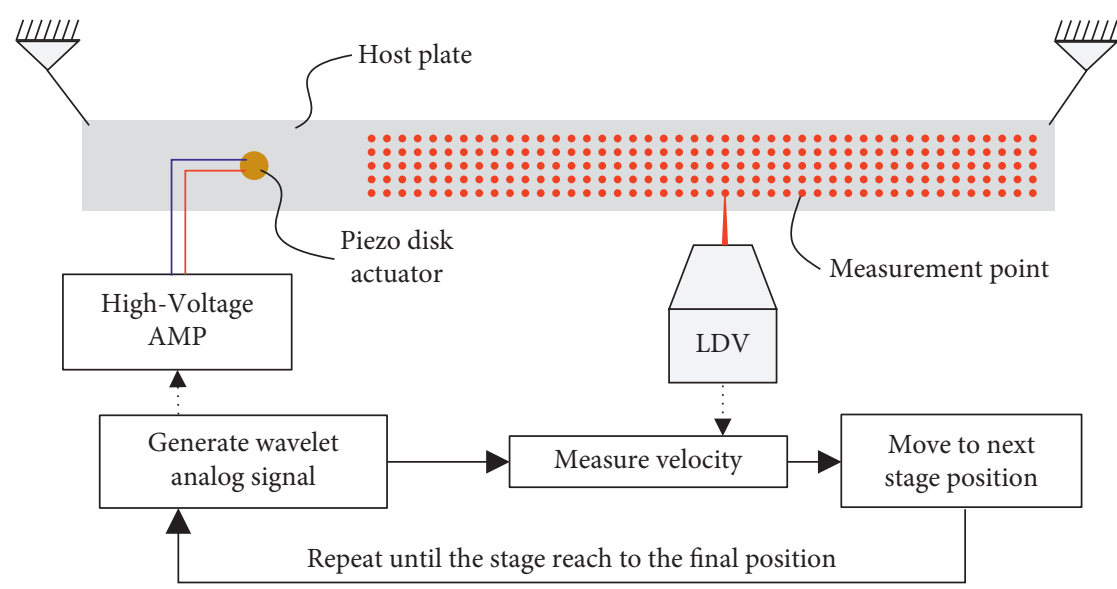

FIgURE 5: Schematic diagram of the experimental setup to measure the wavelength.

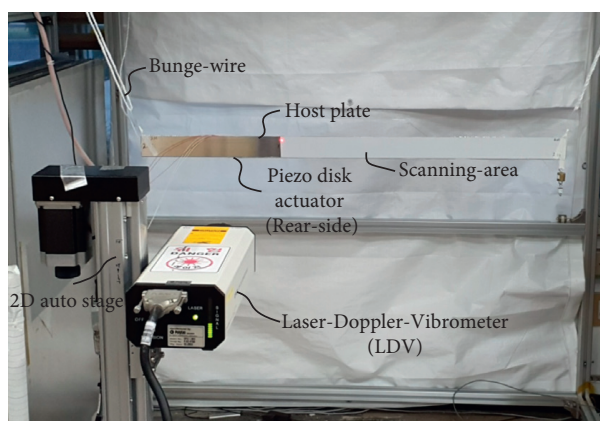

FIGURE 6: Experimental setup for the beam patch test.

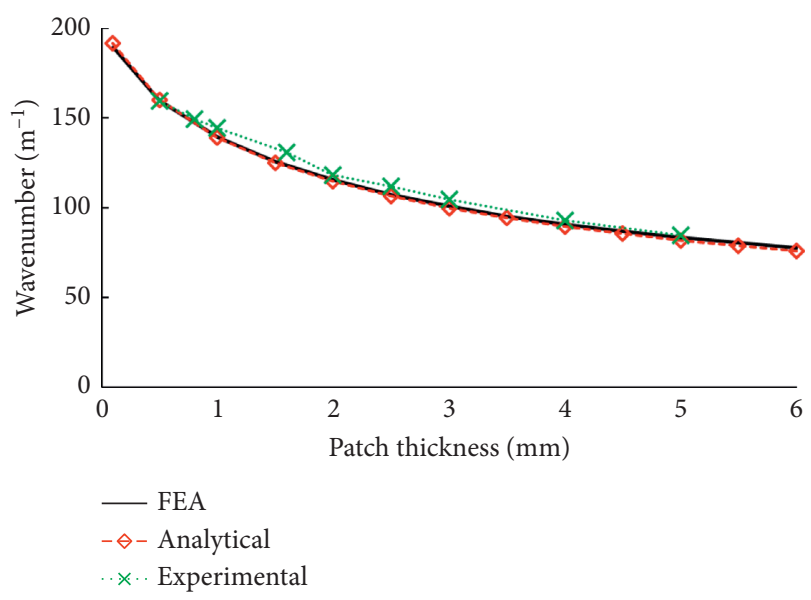

FIgURE 7: Comparison of the FE analysis and theoretical results.

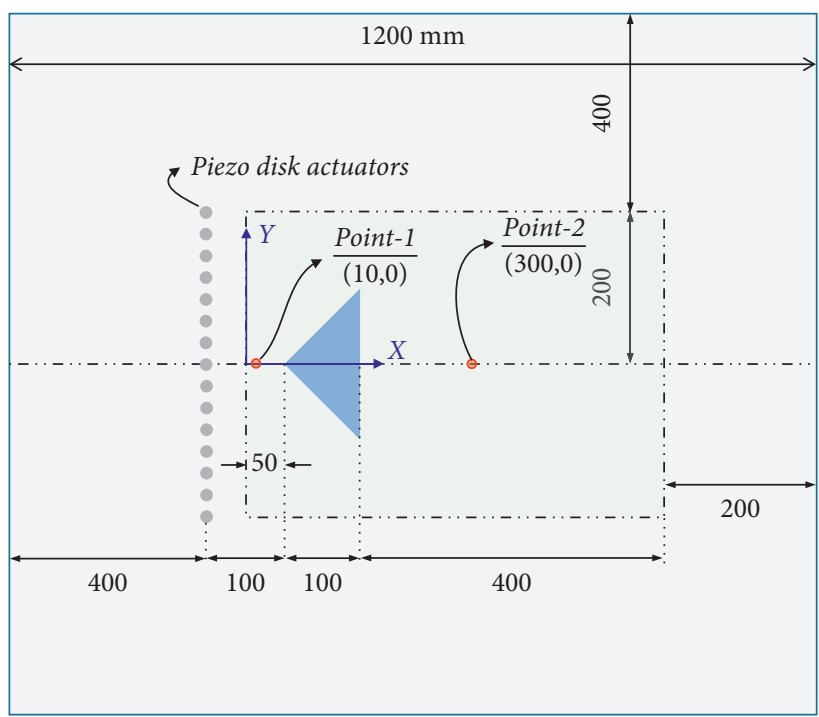

FIgURE 8: Schematic and dimensions of experimental structure.

actuators attached to a thin host-plate made of aluminium; the generated wavelets are refracted when passing through the elastic patch.

In order to refract and defocus the plane wave to reduce the response at the rear of the patch, three simple shaped lenses were considered, as shown in Figure 9: a triangle (prism), a circle (ball lens), and a double-convex lens (DCX lens). The DCX lens is one of the most commonly used lenses in optics [29]. The characteristic dimensions of the patches are taken as the length of the direction perpendicular to the 


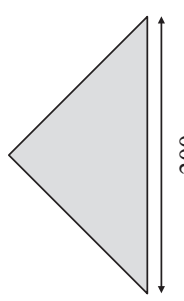

(a)

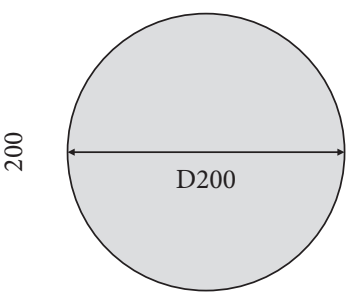

(b)

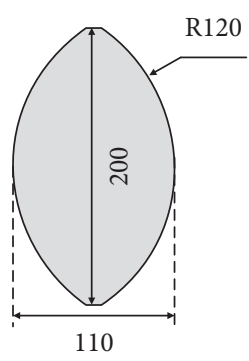

(c)

Figure 9: Dimensions of three different patches: (a) right-angled triangle, (b) circle, and (c) DCX lens.

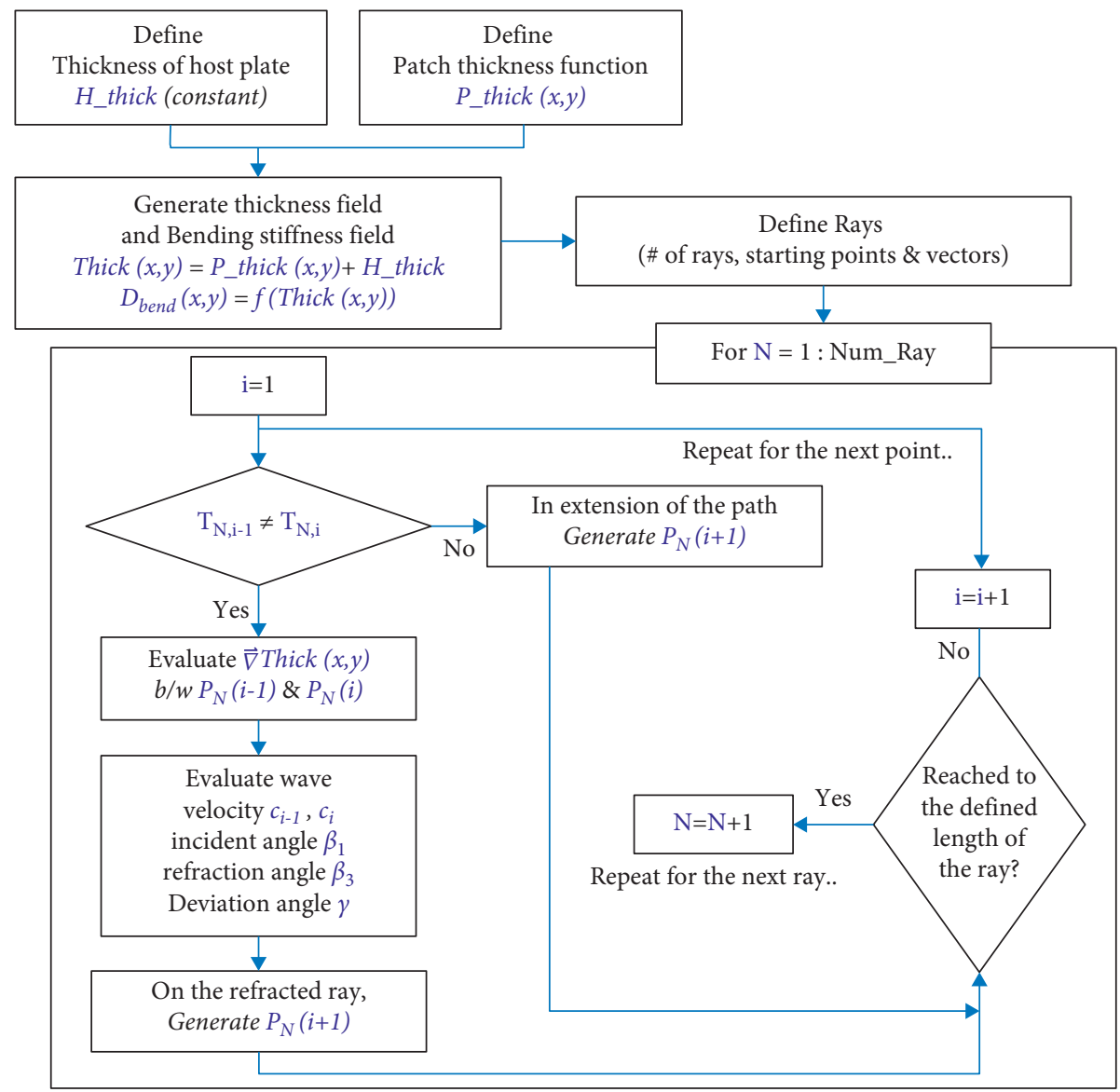

FIGURE 10: The established ray-tracing algorithm.

wave progress; the characteristic lengths of all three patches are set equally to $200 \mathrm{~mm}$. All the patches are made of the same material as the host plate and thickness and are of the same thickness $(1 \mathrm{~mm})$.

4.2. Prediction of Wave Refraction with Ray Tracing. In order to qualitatively predict and compare the shock reduction characteristics at the rear region of each different type of patch, as preliminary evaluation, the ray-tracing method was used. If the incident wavefronts are perfectly plane wave, refraction behaviors of the wavefronts at the boundary of the patches can be effectively simulated by utilizing the raytracing technique. The ray-tracing technique is often used in ocean acoustics and seismology, as well as optics. A common assumption of the ray-tracing techniques in this study is that the bending wave propagates in straight lines through the structure [30]. Therefore, interference and diffraction cannot be calculated by ray tracing. However, the ray-tracing technique is a convenient and powerful way to predict the propagation path of the wavefronts. Figure 10 shows the flowchart of the established ray-tracing algorithm. In the 2dimensional thickness field in $X Y$-plane (see Figure 8), each beam departs from the point source then travels straightly 


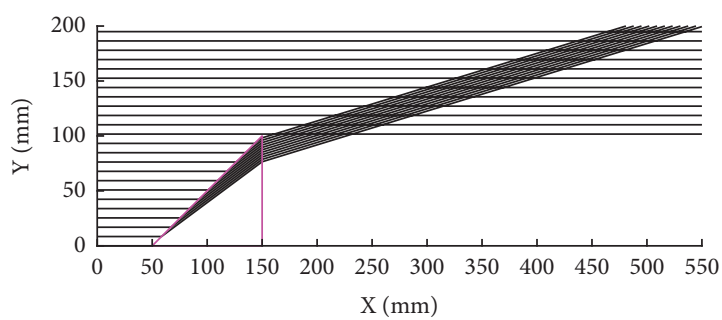

(a)

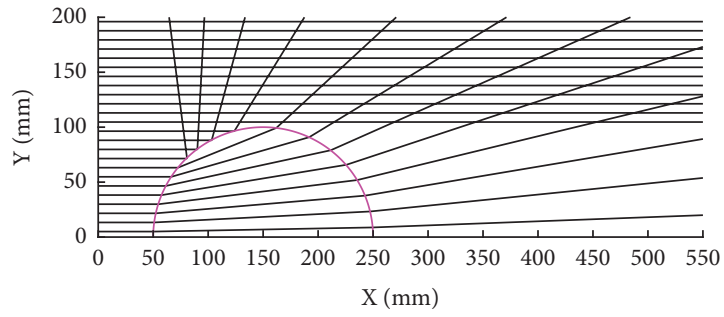

(c)

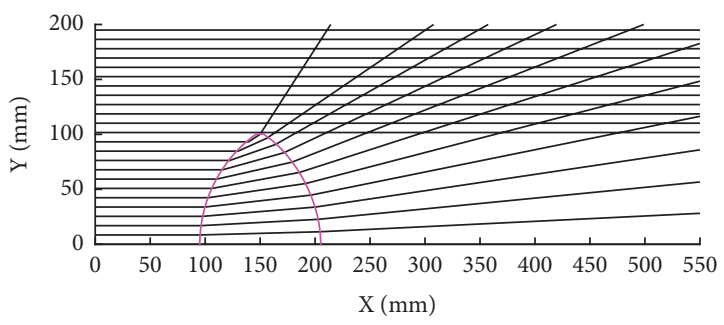

(e)

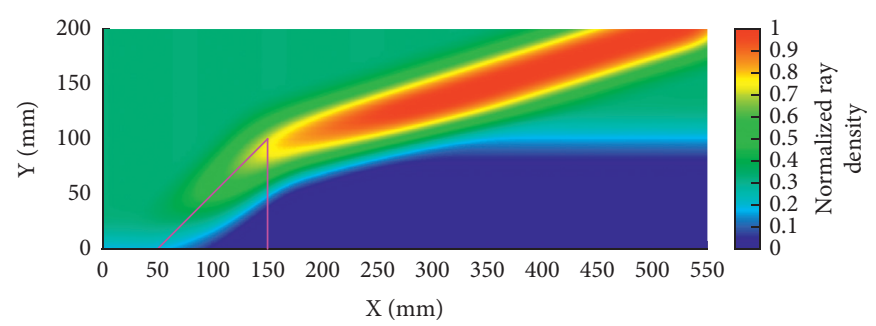

(b)

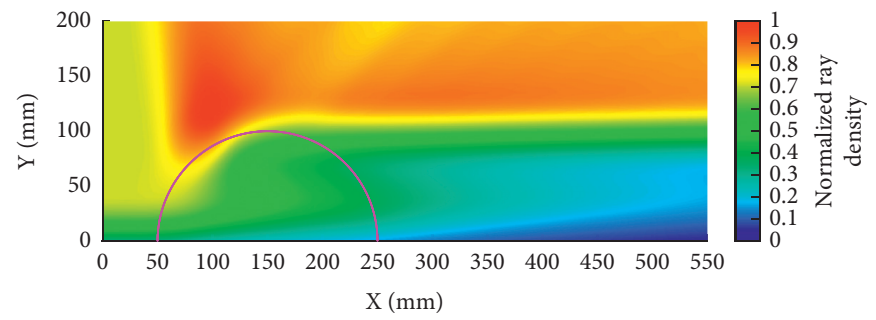

(d)

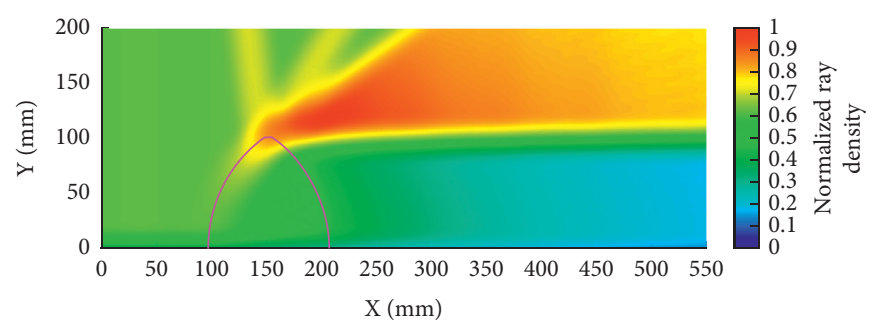

(f)

FIGURE 11: The predicted path of wave transmission by ray-tracing technique.

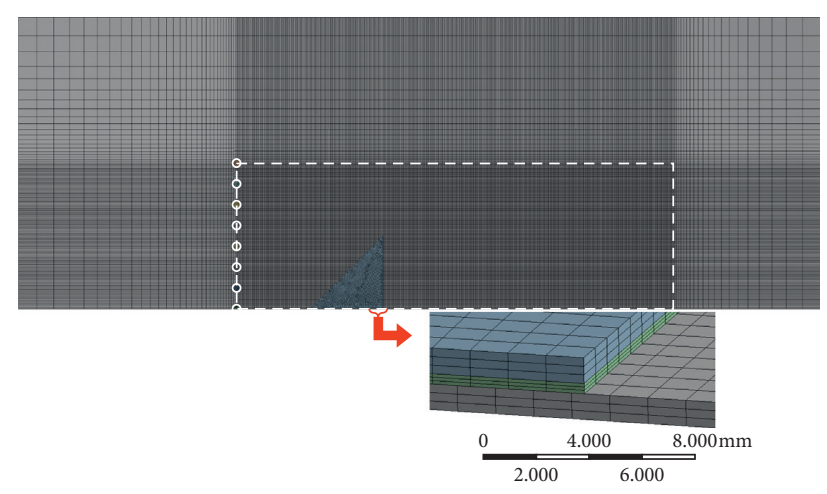

Figure 12: Finite element model for the triangle-patch case.

with discrete small steps which are sufficiently smaller than the length of the patch so that the step size can be negligible. In Figure $10, P_{N}(i)$ is $i$-th point on the $N$-th beam, $T_{N, i}$ denotes thickness at the $i$-th point on the $N$-th beam, and $D_{\text {bend }}$ is the bending stiffness. During travel, when the thickness at the point of step " $i$ " is different from step " $i-1$," the beam direction is adjusted as much as the deviation angle calculated based on the difference of the bending stiffness and the phase velocity between step point " $i-1$ " and step point " $i$."

After completing the beam trajectory calculation, point clouds are generated along each beam, and the density field of the point clouds can be evaluated by the kernel density estimation algorithm. The kernel density estimation provides a simple way of finding structure in data sets without imposing a parametric model [31]. From the calculated density, the high response area and the low response area can be predicted. As the kernel density function, the Epanechnikov kernel [32] as equation (12) was used:

$$
K(u)=\frac{3}{4}\left(1-u^{2}\right),\{|u|<1\} .
$$

The kernel function, $K(u)$, is the weighting function used in nonparametric estimation techniques and also can be 

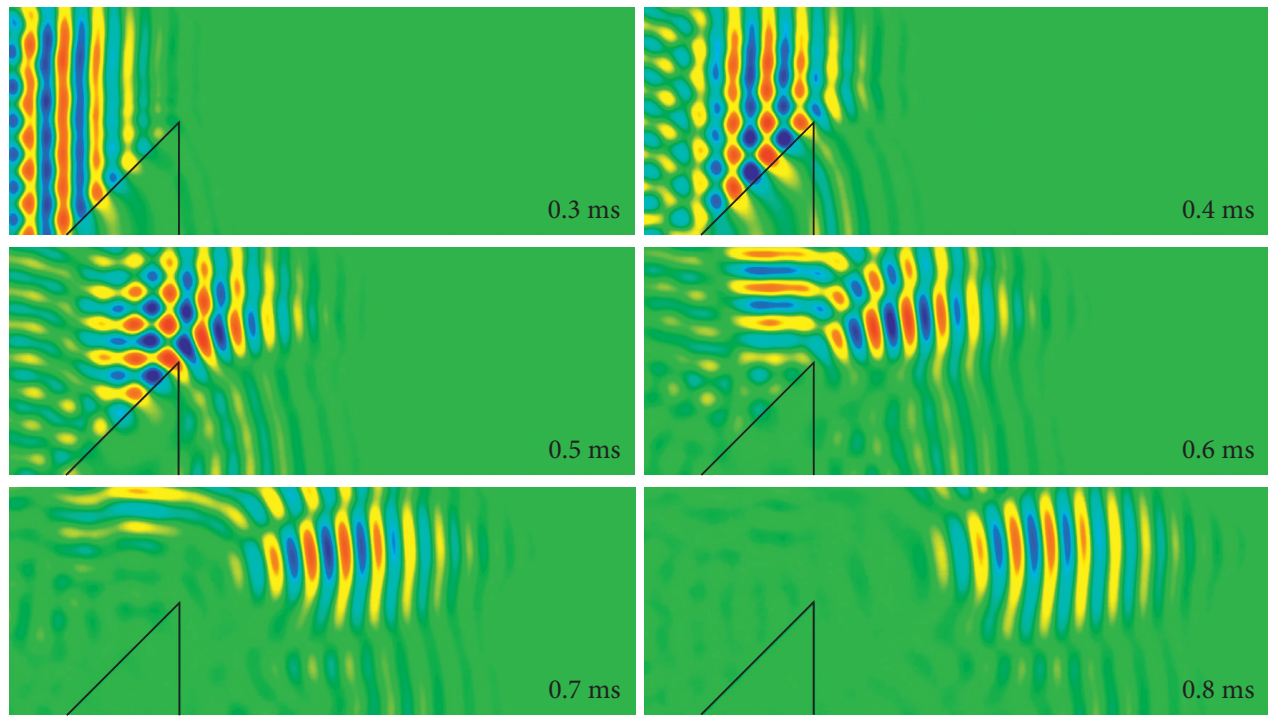

$-1$

0

Figure 13: Numerical simulation of acceleration wavefield for triangle patch.
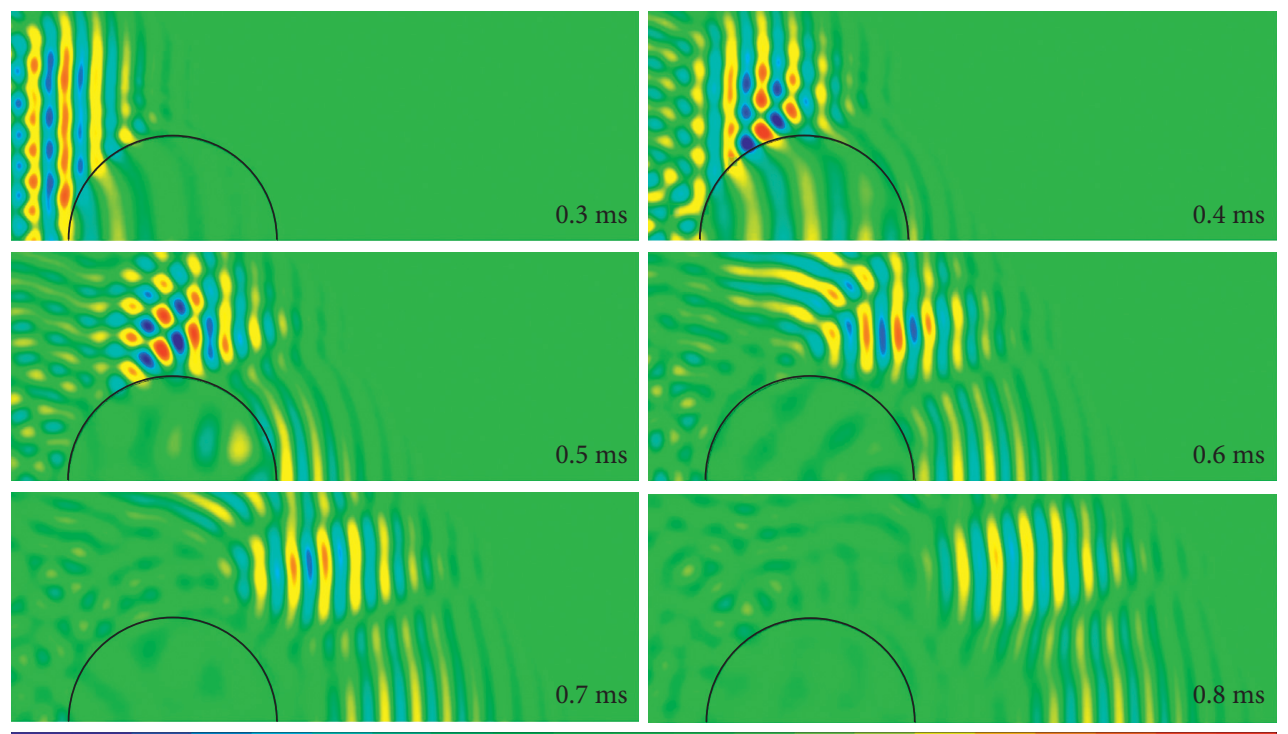

$-1$

0

Figure 14: Numerical simulation of acceleration wavefield for circle patch.

utilized for smoothing data by calculating a weighted average of neighboring observed data. In equation (12), $u$ is the normalized distance from the selected point to the neighboring data, and the normalized distance is defined as

$$
u=\frac{\left\|X_{P}-X\right\|}{R},
$$

where $X_{P}$ and $X$ are coordinate of the selected point and its neighboring data point, respectively, and $R$ is the radius of the window.

Figure 11 shows the calculated beam trajectories (see (a), (c), and (e)) and the normalized density fields (see (b), (d), and (f)) for the case of the triangle, the circle, and the DCXlens patch, respectively. For the area behind the patch, the triangle patch refracts all incident beam with a consistent high-refraction angle, and transmitted beam density is almost zero at this area. On the contrary, the circle patch and lens patch refracts or reflects incident beams with various angles depending on the incident angle of each beam. Significantly, the transmitted beam density at the area behind the lens patch is larger than that of the circle patch because the incident angle of the same beams for the lens patch is smaller than the circle patch, resulting in small refraction angles. 

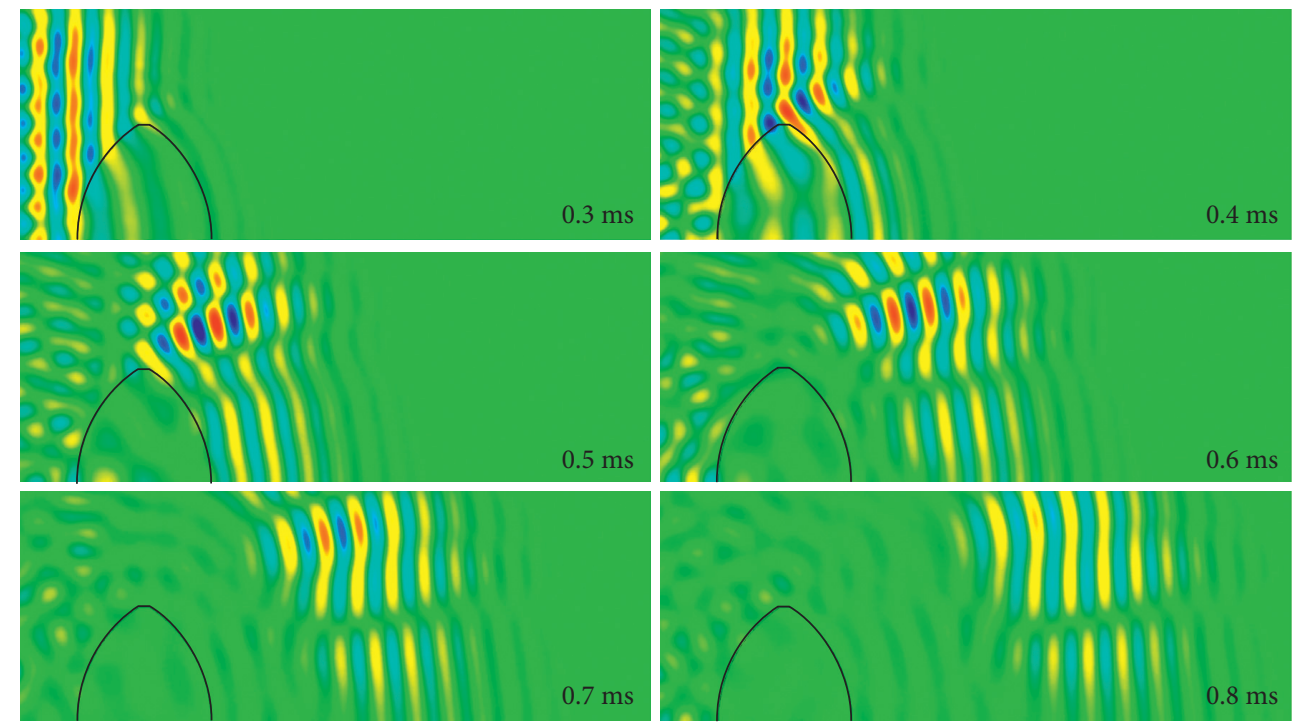

0

Figure 15: Numerical simulation of acceleration wavefield for DCX-lens patch.

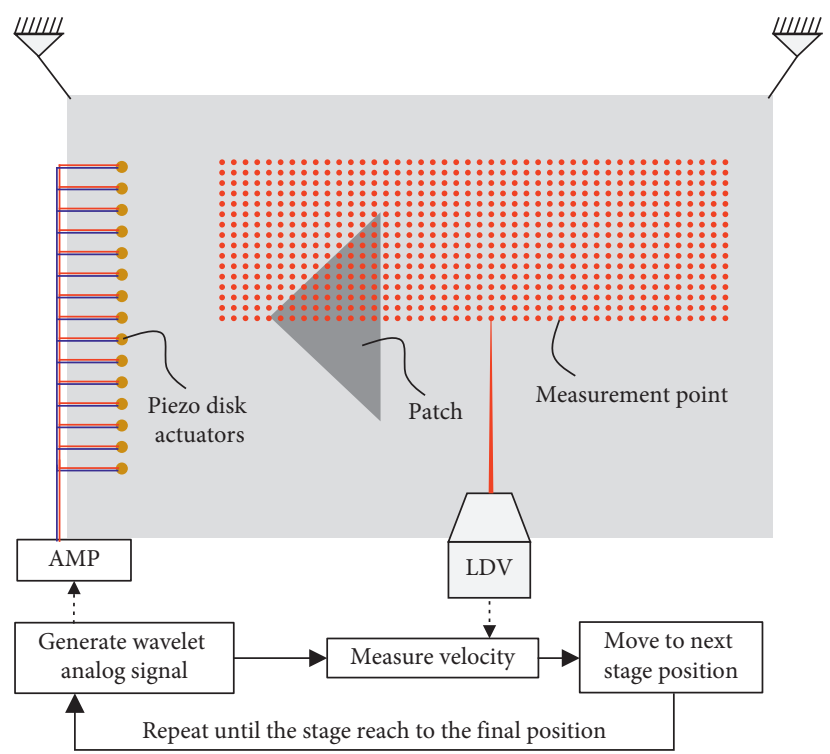

FIGURE 16: Schematic diagram of the experimental setup.

4.3. Numerical Simulation. Wave propagation on a thin plate can be effectively and accurately simulated using the explicit time integration of the finite element method for the Lagrangian approach $[5,33,34]$. A numerical simulation for the wave propagation was conducted using ANSYS Explicit dynamics. The established finite element model using symmetric geometry is shown in Figure 12. A total of 220,000 nodes and 150,000 elements was used. An epoxy layer with an assumed thickness of $0.4 \mathrm{~mm}$ was also considered between the host plate and the patch for the adhesive. The host plate is made of aluminium with $1 \mathrm{~mm}$ thickness; the elastic patch has a right-angled triangle shape for this case and is made of the same material. A total of 15 thin disks with $0.5 \mathrm{~mm}$ thickness and $10 \mathrm{~mm}$ diameter are modelled to mimic the piezo actuators. To generate bending waves, Hanning-windowed $Y$-directional sinusoidal velocity boundary condition with $100 \mathrm{~mm} / \mathrm{s}$ peak and $10 \mathrm{kHz}$ frequency was applied to the disks. The material properties of the aluminium (for the plate and the patch) and the epoxy (for the adhesive) listed in Table 1 were used. An identical analysis setup was applied to the cases of the circle, and DCX lens patches.

Figures 13 to 15 show the propagation and refraction of incident waves by illustrating the normalized acceleration 


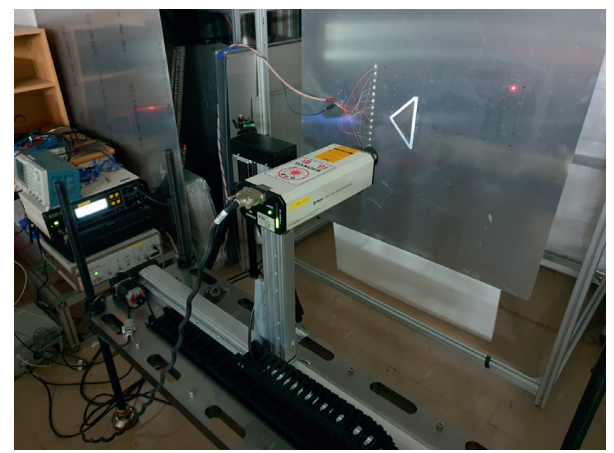

FIGURE 17: Configuration of the built experimental setup.
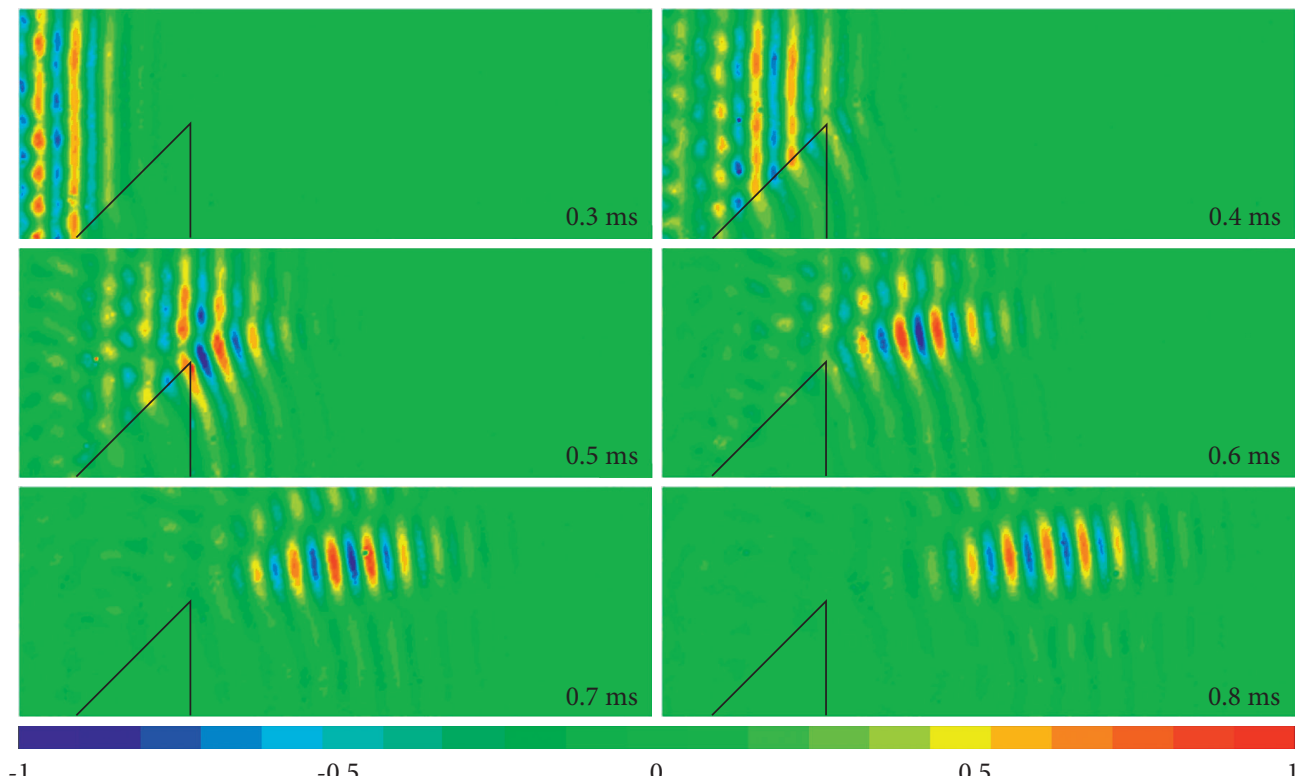

Normalized acceleration

FIGURE 18: Experimentally measured acceleration wavefield for triangle patch.

with the triangle, circle, and DCX-lens-shaped patches, respectively. All results show that the incident waves effectively refracted to the side of each patch; waves transmitted to the area after the patch were significantly reduced. The refracted waves and plane waves on the side of the patches exhibit constructive and destructive interference. Waves generated from the multiple point source gather to create the plane waves; thus, reflected and refracted waves generated through the patch create complex interference fringes. The triangle patch shows lower transmittance than those of other patches because its fixed incident angle of $45^{\circ}$ for the plane waves theoretically causes the largest deviation angle for the boundary of thickness variation. Therefore, the circle patches and the DCX-lens patches have lower reduction rates than those of the triangle patches. In particular, since these two patches have low angles of incidence near the center, which is nearly perpendicular, the waves are reduced only by certain reflections, resulting in relatively high transmission. These trends of the numerical simulation results are consistent with the ray-tracing results discussed in the previous section.

4.4. Experimental Demonstration. Experiments to measure and visualize the wave propagation and refraction were performed. Figure 16 shows a schematic diagram and the built experimental setup. The host plate was made of aluminium with dimensions of $1200 \mathrm{~mm} \times 1200 \mathrm{~mm} \times 1 \mathrm{~mm}$. The patches are made of the same material as the host plate, with equal characteristic dimensions of $200 \mathrm{~mm}$ length in the $Y$-direction. A 1D array of 15-piezoelectric disks, made of PIC255 with $10 \mathrm{~mm}$ diameter and $0.5 \mathrm{~mm}$ thickness, was attached to the host plate. The waveform of the input voltage to the piezo disk actuators was set to a Hanning-windowed sinusoidal signal of three periods, with voltage amplitude from $0 \mathrm{~V}$ to $300 \mathrm{~V}$, amplified using a Trek-623B piezoelectric driver. The velocity histories at each sensing point were measured with a sampling rate of $1 \mathrm{MHz}$ using a National 

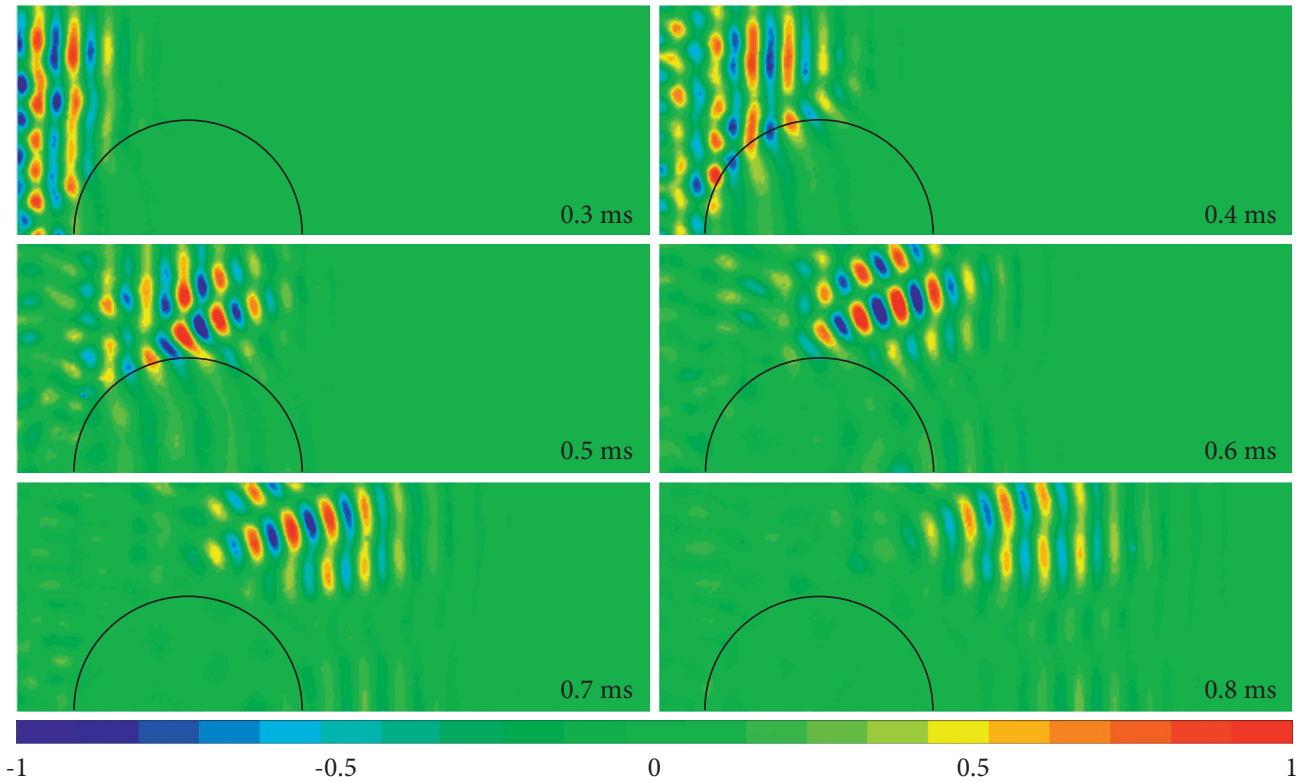

$-0.5$

0

Normalized acceleration

FIgURE 19: Experimentally measured acceleration wavefield for circle patch.
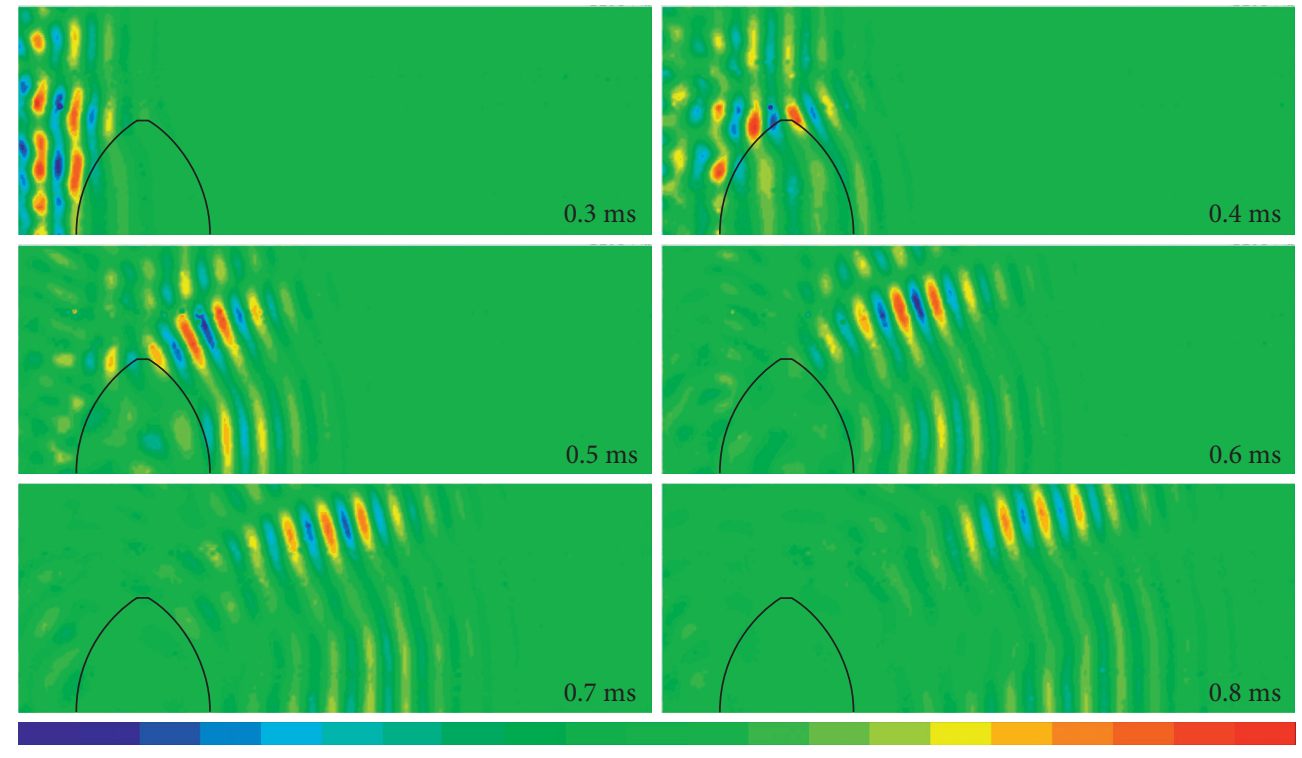

$-1$

$-0.5$

0

0.5

Normalized acceleration

FIGURE 20: Experimentally measured acceleration wavefield for DCX-lens patch.

Instruments PXIe-6366 DAQ board. The scanning area was set as $550 \mathrm{~mm}$ width $\times 200 \mathrm{~mm}$ height with $3 \mathrm{~mm}$ intervals; a total of 12,328 points was measured. Figure 17 shows the described experimental setup for the triangle patch.

Figures 18 to 20 illustrate the normalized acceleration wavefield measured in the experiments. The propagation and the refraction patterns, which are very similar to those of the analysis results, were confirmed. As also confirmed in the numerical simulation results, in the case of the triangle patch, at the incident interface with a critical angle of incidence, some waves are reflected by total internal reflection, and some are refracted and proceed in parallel along the boundary. In this case, the transmittance is low because most waves are completely reflected or refracted across the incident boundary. On the contrary, for the circle patches, incident waves passing through the range of " $Y>70 \mathrm{~mm}$ " approach the patch boundary with a larger incident angle than the critical angle, and so total internal reflection occurs, some incident waves passing through the range of " $Y<70 \mathrm{~mm}$ " are reflected, and some are refracted. 


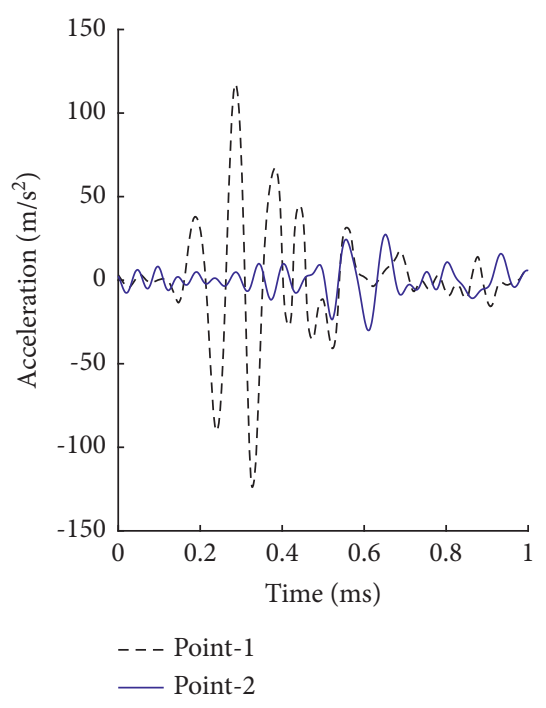

(a)

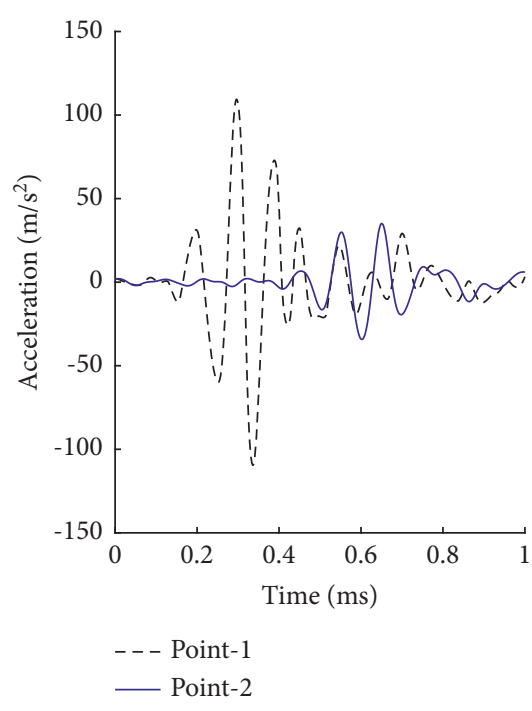

(b)

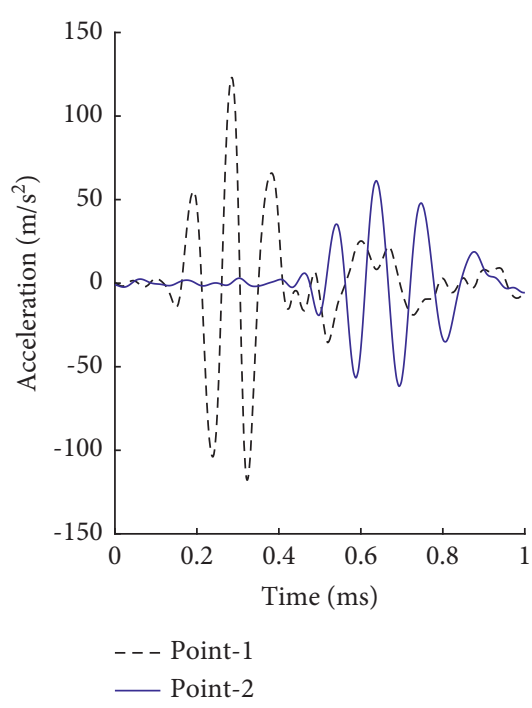

(c)

Figure 21: Acceleration response before and after the patch for cases of (a) triangle, (b) circle, and (c) DCX-lens patches.

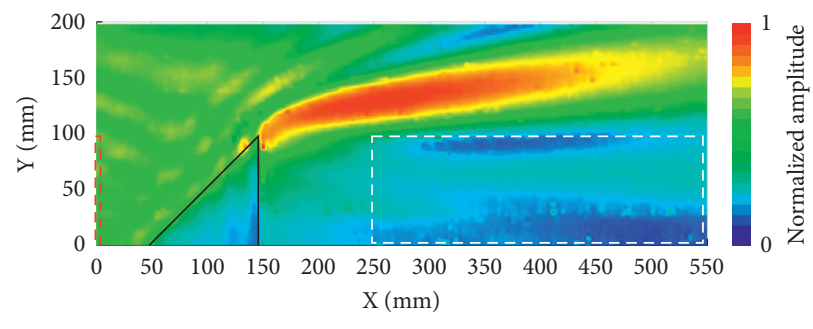

(a)

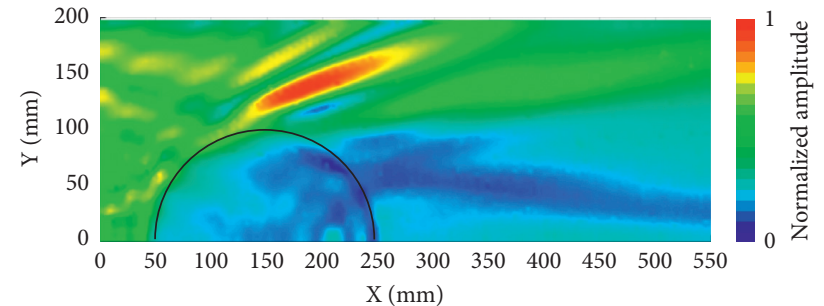

(b)

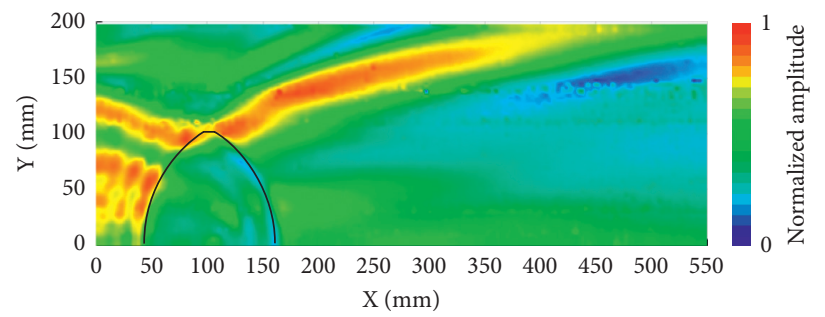

(c)

FIgURE 22: Normalized root mean square value of wavefield for (a) triangle, (b) circle, and (c) DCX-lens-shaped patch.

Figure 21 shows a comparison of the acceleration response at the measurement points (marked in Figure 8) where point -1 is at $(10,0)$ and point -2 at $(300,0)$. The time intervals between the two waveforms, measured at point-1 and point-2, are due to the travel time of the bending waves from point-1 to point- 2 . The shock reduction performance can be evaluated by comparing the amplitude of the waveforms at point- 1 and point- 2 . The triangle patch and the circle patch show a similar amplitude of responses at point2, whereas the DCX-lens patch shows a significantly larger response, thus confirming that the wave reduction effect is relatively low. In order to quantitatively evaluate the reduction performance of each patch, the criterion of reduction ratio was defined and calculated using the root mean square (RMS) value of the acceleration wavefield. Figure 22 displays the normalized root mean square value of the acceleration wavefield for the three different shapes of patches. The triangle patch showed high reduction performance along the central line, while the circle patch and DCX-lens patch showed low reduction rates along the central line. In particular, the DCX-lens patch was found to have low reduction performance overall. In Table 2, the mean RMS value for the area before the patch (red dotted box in Figure 22(a)) and that after the patch (white dotted box in Figure 22(a)) were evaluated at the measurement points of $X=0,0 \leq Y \leq 100$, and $250 \leq X \leq 550,0 \leq Y \leq 100$ (unit: $\mathrm{mm}$ ), respectively. The comparison shows that the triangle patch has better reduction performance than that of the circle and 
TABLE 2: Comparison of wave reduction performance according to the shape of the patch.

\begin{tabular}{|c|c|c|c|c|c|}
\hline \multirow{3}{*}{ Shape of patch } & \multicolumn{2}{|c|}{ Normalized mean RMS $\left(\mathrm{m} / \mathrm{s}^{2}\right.$} & \multirow{3}{*}{ Reduction ratio } & \multirow{3}{*}{ Area of patch $\left(\mathrm{mm}^{2}\right)$} & \multirow{3}{*}{ Efficiency (reduction/area, $\mathrm{mm}^{-1}$ ) } \\
\hline & & & & & \\
\hline & Before patch & After patch & & & \\
\hline Triangle & 1 & 0.185 & 0.815 & 10000.0 & $8.151 \times 10^{-5}$ \\
\hline Circle & 1 & 0.202 & 0.798 & 31415.9 & $2.542 \times 10^{-5}$ \\
\hline DCX-lens & 1 & 0.292 & 0.708 & 17002.2 & $4.167 \times 10^{-5}$ \\
\hline
\end{tabular}

the lens patches, like the results of ray tracing and FEA; furthermore, the reduction per area value of the triangle patch is also the highest. In some applications, such as aerospace structures, the performance-to-weight efficiency of a system is one of the most important specifications. In order of efficiency in that respect, the triangle patch, the DCX-lens patch, and the circle patch show good reduction performances, as listed in Table 2.

\section{Conclusion}

The reduction of structural shocks propagating as elastic waves in thin plate-like structures is an important issue in various engineering applications. In this study, a new shock reduction method of using an elastic patch to defocus bending waves through the refraction of the waves was proposed. Elastic patches of three different shapes (triangle, circle, and double-convex shapes) were considered to refract and reduce transient bending waves.

As a preliminary experiment, the bending wave propagation test on the thin beam assembly, consisting of the host plate, the epoxy adhesive, the patch, was performed for various thicknesses of the patch. The analytically and numerically predicted and experimentally confirmed wavenumber showed a good agreement. To predict shock reduction performance of the patches, the ray tracing and the density estimation algorithm were established. The numerical simulations and the experiments for elastic patches of the three different shapes were performed to identify the propagation and refraction behaviors of the incident plane bending waves. All of the ray tracing, numerical, and experimental results consistently showed that attached patches could effectively reduce passing waves for areas behind patches. The reduction performances of each different shape of the patch were quantitatively evaluated and compared. The reduction ratios, calculated from the root mean square value of the acceleration wavefield, were compared. The triangle patch showed high reduction performance along the central line, while the circle patch and DCX-lens patch showed low reduction rates along the central line. The DCX-lens patch was found to have low reduction performance overall because the incident waves are the plane bending waves. Therefore, the triangle patch can refract all the incident wavefronts with a high-refraction angle. However, in the case of the circle and the DCX lenses, the incident bending waves meet the front edge near the center of those lenses with the low incident angle, almost perpendicular. Comparing the circle and the DCX lenses, the incident angle at the front edge of the DCX lens is lower than the circle lens. Therefore, the reduction performance of the circle patch is better accordingly. In summary, the triangle patch has the best reduction performance; the reduction perarea value of the triangle patch is also the highest. To conclude, it is possible to reduce the transient shock response at certain target areas on various practical structural applications without any degradation of structural stiffness and strength simply by bonding with an elastic patch. Therefore, the proposed method has potential applications in protecting sensitive components such as sensors from intensive shock and noise in plate-like structures in aerospace, automobiles, and constructions.

\section{Data Availability}

The visual illustration data used to support the findings of this study are included in the article.

\section{Conflicts of Interest}

The authors declare that there are no conflicts of interest regarding the publication of this paper.

\section{Acknowledgments}

This work was supported by the National Research Foundation of Korea (NRF) grant funded by the Korea Government (MSIT) (no. NRF-2021R1A2C2004563).

\section{References}

[1] F. J. Fahy, Foundations of Engineering Acoustics, Elsevier Academic Press, London, UK, 2000.

[2] H. K. Jeong, J. H. Han, S. H. Youn, and J. Lee, "Frequency tunable vibration and shock isolator using shape memory alloy wire actuator," Journal of Intelligent Material Systems and Structures, vol. 25, no. 7, pp. 908-919, 2014.

[3] H. K. Jeong, J. Lee, J. H. Han, and N. M. Wereley, "Design of frequency-tunable mesh washer isolators using shape memory alloy actuators," Journal of Intelligent Material Systems and Structures, vol. 27, no. 9, pp. 1265-1280, 2016.

[4] S. H. Youn, Y. S. Jang, and J. H. Han, "Development of a threeaxis hybrid mesh isolator using the pseudoelasticity of a shape memory alloy," Smart Materials and Structures, vol. 20, no. 7, pp. 1-12, 2011.

[5] H. S. Park, D. H. Hwang, J. H. Han, and J. Yang, "Development of shock-absorbing insert for honeycomb sandwich panel," Aerospace Science and Technology, vol. 104, Article ID 105930, 2020.

[6] M. A. Mironov, "Propagation of a flexural wave in a plate whose thickness decreases smoothly to zero in a finite interval," Soviet Physics - Acoustics, vol. 34, no. 3, pp. 318-319, 1988. 
[7] V. Krylov, "Acoustic black holes and their applications for vibration damping and sound absorption," in Proceedings of the International Conference on Noise and Vibration Engineering (ISMA 2012), pp. 933-944, Leuven, Belgium, September 2012.

[8] R. Zhu, X. N. Liu, G. K. Hu, C. T. Sun, and G. L. Huang, "Negative refraction of elastic waves at the deep-subwavelength scale in a single-phase metamaterial," Nature Communications, vol. 5, no. 1, p. 5510, 2014.

[9] M. Dubois, M. Farhat, E. Bossy, S. Enoch, S. Guenneau, and P. Sebbah, "Flat lens for pulse focusing of elastic waves in thin plates," Applied Physics Letters, vol. 103, no. 7, Article ID 071915, 2013.

[10] B. Li, K. Deng, and H. Zhao, "Acoustic guiding and subwavelength imaging with sharp bending by sonic crystal," Applied Physics Letters, vol. 99, no. 5, Article ID 051908, 2011.

[11] A. Darabi, A. Zareei, M.-R. Alam, and M. J. Leamy, "Broadband bending of flexural waves: acoustic shapes and patterns," Scientific Reports, vol. 8, no. 1, Article ID 11219, 2018.

[12] A. Zareei, A. Darabi, M. J. Leamy, and M. R. Alam, "Continuous profile flexural GRIN lens: focusing and harvesting flexural waves," Applied Physics Letters, vol. 112, no. 2, Article ID 023901, 2018.

[13] B. Li, J. Li, S. Yan, W. Yan, and X. He, "Experiment and simulation analysis on noise attenuation of $\mathrm{Al} / \mathrm{MF}$ cylindrical shells," Shock and Vibration, vol. 2017, Article ID 6980501, 8 pages, 2017.

[14] M. Qi, B. Li, S. Yan, Q. Yan, and Q. He, "Simulation analysis of cylindrical shell cavity noise with melamine foam lining," Shock and Vibration, vol. 2021, Article ID 6646596, 9 pages, 2021.

[15] H. Kolsky, Stress Waves in Solids, Dover Publications, New York, NY, USA, 2012.

[16] Z. Tian and L. Yu, "Wavefront modulation and controlling for Lamb waves using surface bonded slice lenses," Journal of Applied Physics, vol. 122, no. 23, Article ID 234902, 2017.

[17] P. Kudela and W. Ostachowicz, "Comparison of Lamb wave focusing performance using wave dispersion-compensated actuation and plano-concave lenses," Journal of Applied Physics, vol. 124, no. 9, Article ID 094901, 2018.

[18] S. Tol, F. L. Degertekin, and A. Erturk, "3D-printed phononic crystal lens for elastic wave focusing and energy harvesting," Additive Manufacturing, vol. 29, Article ID 100780, 2019.

[19] A. Darabi, A. Zareei, M. R. Alam, and M. J. Leamy, "Experimental demonstration of an ultrabroadband nonlinear cloak for flexural waves," Physical Review Letters, vol. 121, no. 17, Article ID 174301, 2018.

[20] K. F. Graff, Wave Motion in Elastic Solids, Dover Publications, New York, NY, USA, 1991.

[21] D. Zenkert, An Introduction To Sandwich Structures, Chameleon Press, London, UK, 1995.

[22] P. Langer, M. Maeder, C. Guist, M. Krause, and S. Marburg, "More than six elements per wavelength: the practical use of structural finite element models and their accuracy in comparison with experimental results," Journal of Computational Acoustics, vol. 25, no. 4, Article ID 1750025, 2017.

[23] D. J. Steinberg, Equation of State and Strength Properties of Selected Materials, Lawrence Livermore National Laboratory, Livermore, CA, USA, 1996.

[24] P. Miranda, A. Pajares, and M. A. Meyers, "Bioinspired composite segmented armour: numerical simulations," Journal of Materials Research and Technology, vol. 8, no. 1, pp. 1274-1287, 2019.
[25] A. Prakash, J. Rajasankar, N. Anandavalli, M. Verma, and N. R. Iyer, "Influence of adhesive thickness on high velocity impact performance of ceramic/metal composite targets," International Journal of Adhesion and Adhesives, vol. 41, pp. 186-197, 2013.

[26] Physik Instrumente (PI) GmbH \& Co. KG, "Piezoelectric materials," 2021, https://www.piceramic.com/en/products/ piezoelectric-materials/.

[27] Advanced Energy Industries, Inc., "High voltage productsTrek 623B," 2021, https://www.advancedenergy.com/ products/high-voltage-products/high-voltage-amplifiers/ standard-high-speed-amplifiers/trek-623b/.

[28] National Instruments Corp., "PXI multifunction I/O module manual-PXIe-6366," 2021, https://www.ni.com/ documentation/en/pxi-multifunction-io-module/latest/pxie6366/overview/\#.

[29] J. Shamir, Optical Systems and Processes, SPIE Press, Bellingham, WA, USA, 1999.

[30] A. H. Andersen and A. C. Kak, "Digital ray tracing in twodimensional refractive fields," Journal of the Acoustical Society of America, vol. 72, no. 5, pp. 1593-1606, 1982.

[31] M. P. Wand and M. C. Jones, Kernel Smoothing, CRC Press, Boca Raton, FL, USA, 1994.

[32] V. A. Epanechnikov, "Non-parametric estimation of a multivariate probability density," Theory of Probability and its Applications, vol. 14, no. 1, pp. 153-158, 1969.

[33] J. Lee, D. H. Hwang, J. K. Jang et al., "Pyroshock prediction of ridge-cut explosive bolts using hydrocodes," Shock and Vibration, vol. 2016, Article ID 1218767, 14 pages, 2016.

[34] J. Lee, D. H. Hwang, and J. H. Han, "Study on pyroshock propagation through plates with joints and washers," Aerospace Science and Technology, vol. 79, pp. 441-458, 2018. 